Article

\title{
Prognostic Roles of BRAF, KIT, NRAS, IGF2R and SF3B1 Mutations in Mucosal Melanomas
}

\author{
Joanna P. Wróblewska ${ }^{1}\left(\mathbb{D}\right.$, Dora Dias-Santagata ${ }^{2} \mathbb{D}$, Adam Ustaszewski ${ }^{3}$,, Cheng-Lin Wu ${ }^{4}(\mathbb{D}$, \\ Masakazu Fujimoto ${ }^{5}$, M. Angelica Selim ${ }^{6}$, Wojciech Biernat ${ }^{7}{ }^{\mathbb{D}}$, Janusz Ryś ${ }^{8}$, Andrzej Marszalek ${ }^{1}$ \\ and Mai P. Hoang $2, * \mathbb{D}$
}

check for

updates

Citation: Wróblewska, J.P.;

Dias-Santagata, D.; Ustaszewski, A.;

Wu, C.-L.; Fujimoto, M.; Selim, M.A.;

Biernat, W.; Ryś, J.; Marszalek, A.;

Hoang, M.P. Prognostic Roles of

BRAF, KIT, NRAS, IGF2R and SF3B1

Mutations in Mucosal Melanomas.

Cells 2021, 10, 2216. https://doi.org/

10.3390/cells10092216

Academic Editors: Barbara Stecca and Laura Poliseno

Received: 3 August 2021

Accepted: 25 August 2021

Published: 27 August 2021

Publisher's Note: MDPI stays neutral with regard to jurisdictional claims in published maps and institutional affiliations.

Copyright: (c) 2021 by the authors. Licensee MDPI, Basel, Switzerland. This article is an open access article distributed under the terms and conditions of the Creative Commons Attribution (CC BY) license (https:/ / creativecommons.org/licenses/by/ $4.0 /)$.
1 Department of Pathology, Poznan University Medical Sciences and Greater Poland Cancer Center, 61-701 Poznan, Poland; Joanna.wroblewska@wco.pl (J.P.W.); amars@ump.edu.pl (A.M.)

2 Department of Pathology, Massachusetts General Hospital and Harvard Medical School, Boston, MA 02114, USA; ddiassantagata@mgh.harvard.edu

3 Institute of Human Genetics, Polish Academy of Sciences, 60-479 Poznan, Poland; adam.ustaszewski@igcz.poznan.pl

4 Department of Pathology, National Cheng Kung University Hospital, College of Medicine, National Cheng Kung University, Tainan 70403, Taiwan; wujl.towalkwithwings@gmail.com

5 Department of Pathology, Kyoto University Hospital, Kyoto 606-8507, Japan; fujimasa@kuhp.kyoto-u.ac.jp

6 Department of Pathology, Duke University Medical Center, Durham, NC 27710, USA; angelica.selim@duke.edu

7 Department of Pathology, Medical University of Gdansk, 80-210 Gdansk, Poland; biernat@gumed.edu.pl

8 Department of Pathology, Maria Sklodowska-Curie National Research Institute of Oncology, 31-115 Cracow Branch, Poland; z5rys@cyf-kr.edu.pl

* Correspondence: mhoang@mgh.harvard.edu

Abstract: Background: The prognostic value of commonly recurrent mutations remains unclear in mucosal melanomas. Methods: Clinicopathologic parameters of 214 cases of mucosal melanomas diagnosed in 1989-2020 in several clinical institutions were analyzed. NRAS, KIT, BRAF, IGF2R and $S F 3 B 1$ mutational analyses by Sanger sequencing and next generation sequencing-based assay were performed in a subset of cases. Results: Of the triple (BRAF, NRAS, NF1)-negative cases, APC, KIT and $K R A S$ are detected mainly in sinonasal, vulvovaginal and anorectal melanomas, respectively. NRAS, KIT, BRAF, IGF2R and SF3B1 mutations are detected in 19\% (37/198), 22\% (44/197), 12\% $(25 / 201), 16 \%(22 / 138)$ and $15 \%(20 / 133)$ of cases, respectively. In univariate analyses, advanced stage $(p=0.016), 65$ years or older $(p=0.048)$ and presence of ulceration $(p=0.027)$ are significantly correlated with worse overall survival (OS), respectively. NRAS mutation significantly correlates with worse OS ( $p=0.028)$ and worse melanoma-specific survival (MSS) $(p=0.03)$ for all cases of mucosal melanomas. In multivariate analyses, NRAS mutation remains as an independent predictor of worse OS $(p=0.036)$ and worse MSS $(p=0.024)$. Conclusion: NRAS mutation is a predictor of worse survival, independent of stage in mucosal melanomas. The significance of frequently mutated $I G F 2 R$ in mucosal melanomas remains unclear.

Keywords: mucosal melanoma; vulvovaginal; sinonasal; anorectal; NRAS; KIT; BRAF; SF3B1; IGF2R

\section{Introduction}

Mucosal melanomas encompass primary melanomas of the female genital tract (vulvar and vaginal melanoma), male genital tract (penile melanoma), head and neck region (sinonasal and oral melanoma), conjunctiva, upper gastrointestinal tract (esophagus, stomach, intestine), anorectal melanoma and urinary tract (urethra, urinary bladder) [1]. While previously considered to be a subtype of ocular melanoma, recent studies have shown that conjunctival melanomas have a similar tumorigenetic pathway as mucosal melanomas. Although mucosal melanomas are rare and constitute approximately $1.4 \%$ of all melanomas, the prognosis of patients with mucosal melanoma is poorer in comparison to cutaneous melanomas. 
In line with prognosis, genetic alterations occurring in mucosal melanoma are different than those of cutaneous wild-type melanoma (BRAF, NRAS and NF1 negative) [2,3]. The frequency of frequently mutated genes are as follows: NRAS (14-30\%), BRAF (5-16\%), NF1 (16\%), KIT (5-15\%), SF3B1 (12\%), TP53 (8.9\%), SPRED1 (7\%), ATRX (6\%) and CHD8 (4\%) [4-10]. Recently, IGF2R mutation was documented in $32 \%$ of 41 mucosal melanomas in comparison to $6 \%$ of 48 cutaneous melanomas [10].

In a series of 444 mucosal melanomas from a European population investigated by Sanger sequencing, NRAS, KIT and BRAF mutations were evenly distributed across the different mucosal melanoma subgroups [8]. The prognostic role of these commonly recurrent mutations in mucosal melanomas has only been studied in some series $[6,7,11,12]$. In a large series of 706 mucosal melanomas, KIT and BRAF mutational status did not correlate with overall survival (OS); however, NRAS was not analyzed in this series [6]. Correlation between NRAS, BRAF and KIT mutations and survival was not observed in prior series of sinonasal melanomas [7,12]. KIT mutation has been reported to be a marker of better progression-free survival in vulvar melanomas [11].

Although mutational status provides potential therapeutic targets, prognostic value of commonly recurrent mutations remains unclear in mucosal melanomas. In this study, we analyzed the prognostic role of NRAS, KIT, BRAF, IGF2R and SF3B1 mutations in a series of mucosal melanomas.

\section{Materials and Methods}

The study was approved by Institutional Review Boards. Mucosal melanomas diagnosed between 1989 and 2020 were retrieved from the pathology archives of several clinical institutions in Japan, Poland, Spain, Taiwan and the United States. A total of 214 melanocytic tumors from 214 patients which were diagnosed with primary vulvar (73), vaginal (4), sinonasal (93), anorectal (31), conjunctival (8), urethral (1) and penile (4) melanomas were included in the study. Melanomas on the vulvar hair bearing skin are excluded. NRAS, KIT and BRAF mutational results of 72 sinonasal, 27 vulvar and 4 vaginal melanomas; and $S F 3 B 1$ results of 72 sinonasal melanomas from prior studies were included [11-13].

\subsection{Clinical Findings and Histologic Features}

The histopathologic diagnoses and following features were assessed by the contributing pathologists and confirmed by the corresponding author (MPH): ulceration, mitotic rate (per squared millimeters), lymphovascular invasion, and perineural invasion. The following data were extracted from medical records: age of the patients, lesion site, date of biopsy, disease status over time and at last follow-up (recurrence, metastasis) and any treatment.

\subsection{Molecular Analyses}

Sanger sequencing and next generation sequencing (NGS) were performed on subset of cases as outlined in Table 1. Deoxyribonucleic acid (DNA) was extracted from formalinfixed paraffin-embedded tumors. For Sanger sequencing NRAS exons 1 and 2; KIT exons 9, 11, 13 and 17; BRAF exon 15; SF3B1 codons 625 and 666 of exon 14, codon 700 of exon 15; and IGF2R exons 2, 6, 8, 16, 43 and 46 were amplified by polymerase chain reaction (PCR) with specific primers (Table S1) [12]. 
Table 1. Summary of cases analyzed by next generation sequencing (NGS) and Sanger sequencing.

\begin{tabular}{ccccccc}
\hline Melanoma & BRAF NRAS & \multicolumn{5}{c}{ Sanger } \\
\cline { 3 - 7 } Subtype & KIT NGS & BRAF & NRAS & KIT & SF3B1 & IGF2R \\
\hline Vulvovaginal & 32 & 33 & 29 & 28 & 37 & 41 \\
\hline Sinonasal & 13 & 80 & 80 & 80 & 80 & 80 \\
\hline Anorectal & 17 & 13 & 14 & 14 & 15 & 16 \\
\hline Conjunctival & 8 & 0 & 0 & 0 & 0 & 0 \\
\hline Penile & 4 & 0 & 0 & 0 & 0 & 0 \\
\hline Urethra & 1 & 0 & 0 & 0 & 1 & 1 \\
\hline Total & 75 & 126 & 123 & 122 & 133 & 138 \\
\hline
\end{tabular}

For IGF2R variant calling, raw genomic sequence data were obtained from NCBI (National Center for Biotechnology Information) Sequence Read Archive under Bioproject number PRJNA379027.10. The raw data consisted of 41 mucosal melanoma samples and were quality checked using FastQC software and mapped to the reference genome (GRCh38/hg38) using BWA-MEM [14]. Further analysis, including duplicated reads marking, base quality scores recalibration and eventually variant calling, was performed according to GATK best practices pipeline [15]. The preprocessed cohorts of variants were filtered using SnpSift [16]. Only detected variants with a high score for predicted pathogenicity were chosen for verification in mucosal melanoma samples cohort (Table S2).

NGS-based molecular tests were performed on 75 cases. Twenty-six cases were from prior studies $[11,13]$. Single nucleotide variants (SNV) and small insertion/deletions (indel) in genomic DNA were detected using Anchored Multiplex Polymerase chain reaction (PCR) by NGS (Table S3) [17]. A sequencing library targeting hotspots and exons in 99 cancer genes was generated using two hemi-nested PCRs. Using BWA-MEM Illumina MiSeq, $2 \times 151$ base paired-end sequencing results were aligned to the hg19 human genome reference [14]. For indel variant and SNV detection, a laboratory-developed insertion/deletion analysis algorithm and MuTech were used, respectively [18].

\subsection{Statistical Analysis}

The statistical associations between mutation of NRAS, BRAF, KIT, SF3B1, IGF2R and clinicopathologic features (patient's age, stage, ulceration, mitotic index, lymphovascular invasion, perineural invasion, progression, recurrence, metastasis and death) were evaluated by Fisher's exact tests. The number of months from diagnosis to development of locally recurrent or metastatic disease in the lymph nodes or distant organs was defined as progression-free survival (PFS). In patients with disease progression, time of death was equated to melanoma-related death. The number of months from initial diagnosis to patient's death by any cause and related to melanoma were defined as overall survival (OS) and melanoma-specific survival (MSS), respectively. Kaplan-Meier plots and log-rank tests were done to visually assess the differences in OS, MSS and PFS between subgroups. Univariate analyses were performed with the Cox proportional hazards model. All covariates with $p<0.05$ were included in the multivariate Cox proportional hazard model. All statistical analyses were done using the $\mathrm{R}$ statistical package [19]. A two-tailed $p$ of less than or equal to 0.05 was considered to be statistically significant.

\section{Results}

The study included 214 patients. The age of the patients ranged from 20 to 91 years (median, 65 years). The follow-up (FU) for all patients ranged from 0 to 233 months (median, 21 months). Progression (local recurrence and/or metastasis) developed in $156 / 214(73 \%)$ patients. Metastases developed in 121/207 (58\%) patients, with distant metastases seen in 88/207 (43\%), with lung and/or liver being the most common metastatic 
sites. Death was documented in 117/214 (55\%) patients. The patients were categorized into stage I/II versus stage III/IV to reflect whether metastasis was documented at time of diagnosis, due to incomplete data such as tumor size and tumor thickness in some cases, such as sinonasal melanomas. There were 168 patients with stage I/II, 45 with stage III/IV and 1 without known stage. There were no survival differences among the patients with vulvovaginal, sinonasal and anorectal melanomas (Figure 1A). Patients from Europe (42 patients; median FU, 11 months) have better OS ( $p=0.01)$ in comparison to those from North America (124 patients; median FU, 24 months) and Asia (44 patients; median FU, 20 months) (Figure 1B).

A

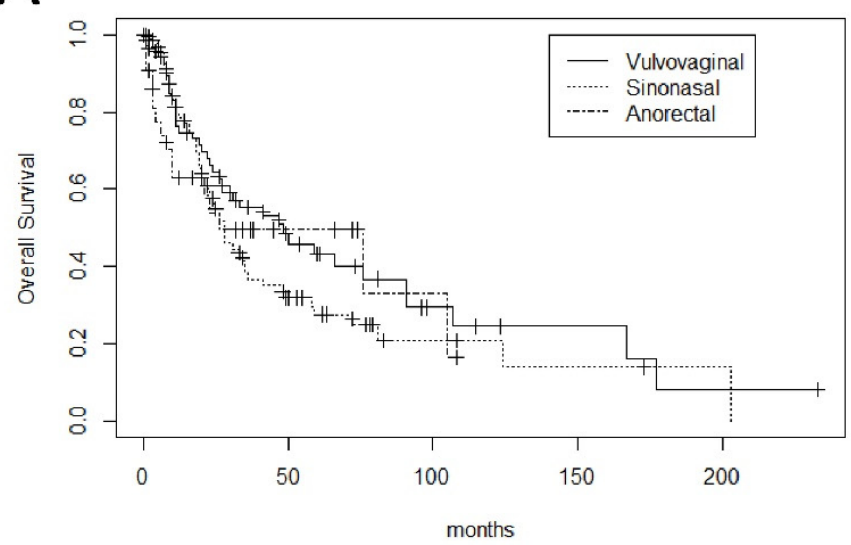

B

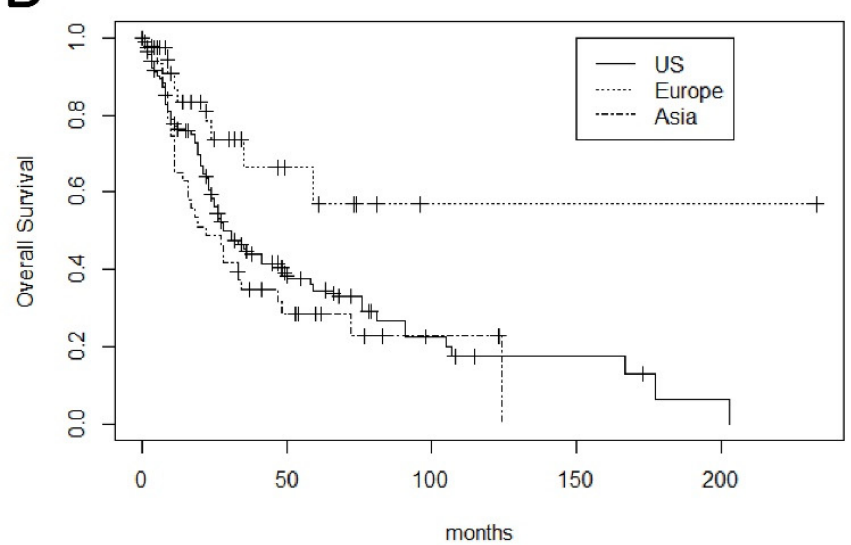

Figure 1. (A) Kaplan-Meier curves demonstrate no significant correlation between overall survival and anatomic sites ( $\log$-rank $p=0.3$ ); (B) Kaplan-Meier curves demonstrate a significant correlation between overall survival and geographic areas $(\log -\operatorname{rank} p=0.01)$.

Ulceration, lymphovascular invasion and perineural invasion were noted in 144/202 (71\%), 41/209 (20\%) and 26/209 (12\%) cases, respectively. The number of mitoses identified per millimeter squared ranged from 0 to 100 (median, 7). Except for the correlation between $N R A S$ and IGF2R mutation and presence of ulceration $(p=0.031)$ and lymphovascular invasion $(p=0.03)$, respectively, there were no associations between NRAS, BRAF, KIT, $S F 3 B 1$ and IGF2R mutations and clinicopathologic features.

NRAS, KIT, BRAF, IGF2R and SF3B1 mutational status were known in 198, 197, 201, 138 and 133 cases, respectively. NRAS, KIT, BRAF, IGF2R and SF3B1 mutations were detected in 19\% (37/198), 22\% (44/197), 12\% (25/201), 16\% (22/138) and 15\% (20/133) cases, respectively (Figure 2). Mutations of codons 61 and 12/13 of NRAS were detected in $62 \%$ and $38 \%$ of mutated cases, respectively. KIT L576P mutation was seen in $33 \%$. BRAF V600E mutation was present in $72 \%$ of mutated cases. Mutations involving codon 625 of SF3B1 was detected in $80 \%$ of mutated cases (Figure 2). 


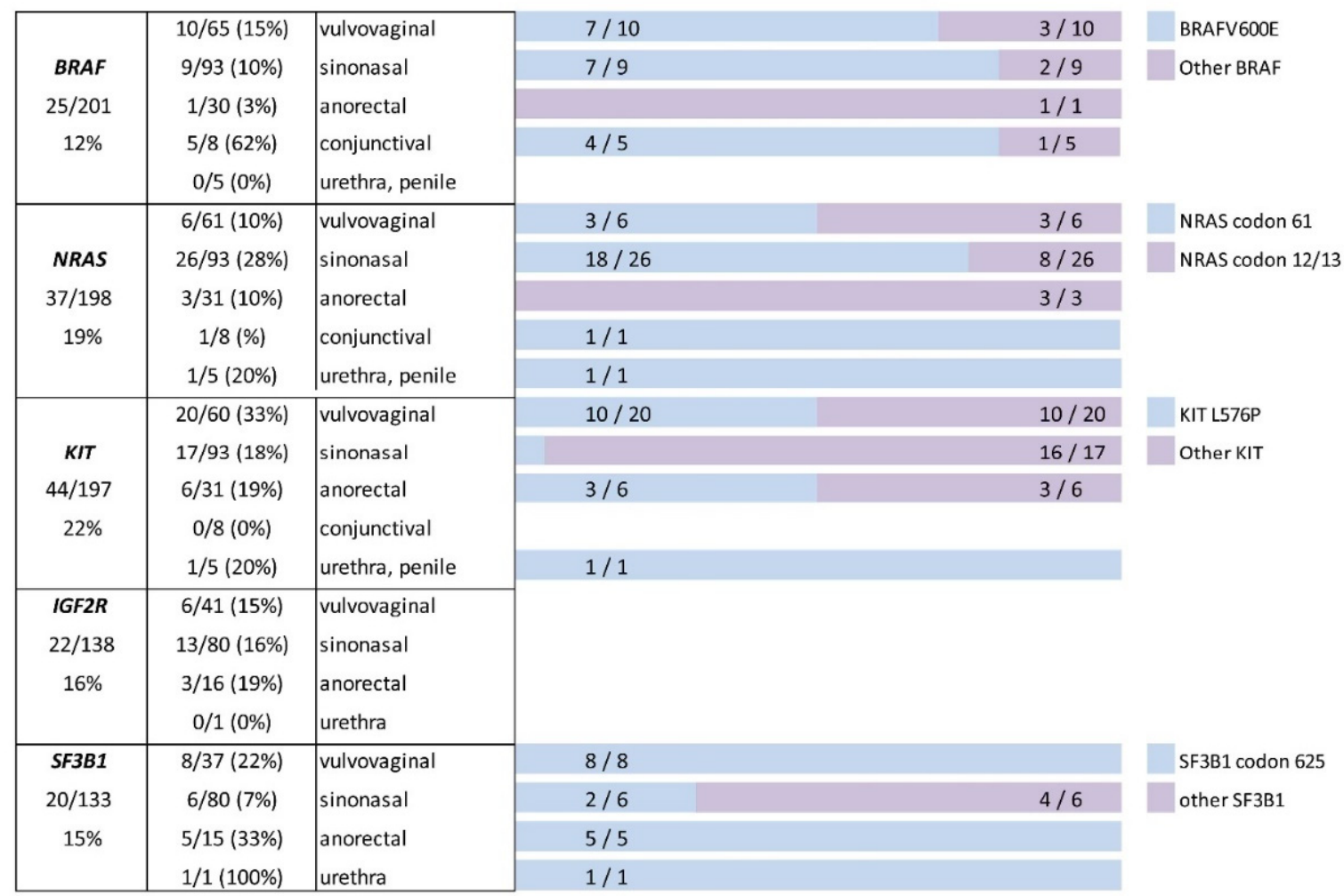

Figure 2. Summary of the molecular analyses. BRAF V600E, NRAS codon 61, KIT L576P and SF3B1 codon 625 are the most frequently detected mutations.

The NGS results of 49 mucosal melanomas (6 vulvar, 17 anorectal, 13 sinonasal, 8 conjunctival, 1 urethral and 4 penile), together with the published results of 26 vulvovaginal melanomas, are summarized in Figure $3[11,13]$. BRAF, KIT and NRAS were the most common recurrent mutations, seen more frequently in vulvovaginal and sinonasal melanomas in comparison to anorectal melanomas. In addition, mutations affecting the APC, ATM, ATRX, CDH1, KRAS, NF1, NF2, PIK3R1, TSC2, TP53 and TERT promoter regions were noted. Of the triple (BRAF, NRAS, NF1)-negative cases, APC, KIT and KRAS were detected mainly in sinonasal, vulvovaginal and anorectal melanomas, respectively. Copy number variants, including loss of CDKN2A and gain of KIT, CDK4 and MYC, were frequently seen in mucosal melanomas.

Univariate analyses are performed for the following variables: NRAS, KIT, BRAF, SF3B1 and IGF2R mutation; stage; age; ulceration; mitoses; perineural invasion; lymphovascular invasion; and adjuvant therapy. Advanced stage ( 3 or $4, p=0.018$ ), age older than 65 years $(p=0.036)$ and presence of ulceration $(p=0.028)$ were significantly correlated with worse OS, respectively (Table S4). Whether the patient had received adjuvant therapy affected only PFS but not OS or MSS (Table S4). No significant correlation was observed between adjuvant therapy and overall survival. NRAS mutation significantly correlated with worse OS $(p=0.026)$ (Figure 4A) and worse MSS $(p=0.031)$ for all cases of mucosal melanomas. When stratified into subgroups, KIT mutation significantly correlated with improved PFS ( $p=0.0021$ ) for vulvovaginal melanomas (Figure 4B) and BRAF mutation with worse PFS for sinonasal melanomas $(p=0.0045)$ (Figure $4 \mathrm{C}$ and Table S4). No significant correlation with survival was seen for IGF2R and SF3B1 mutations for all cases as well as for individual subgroups. 


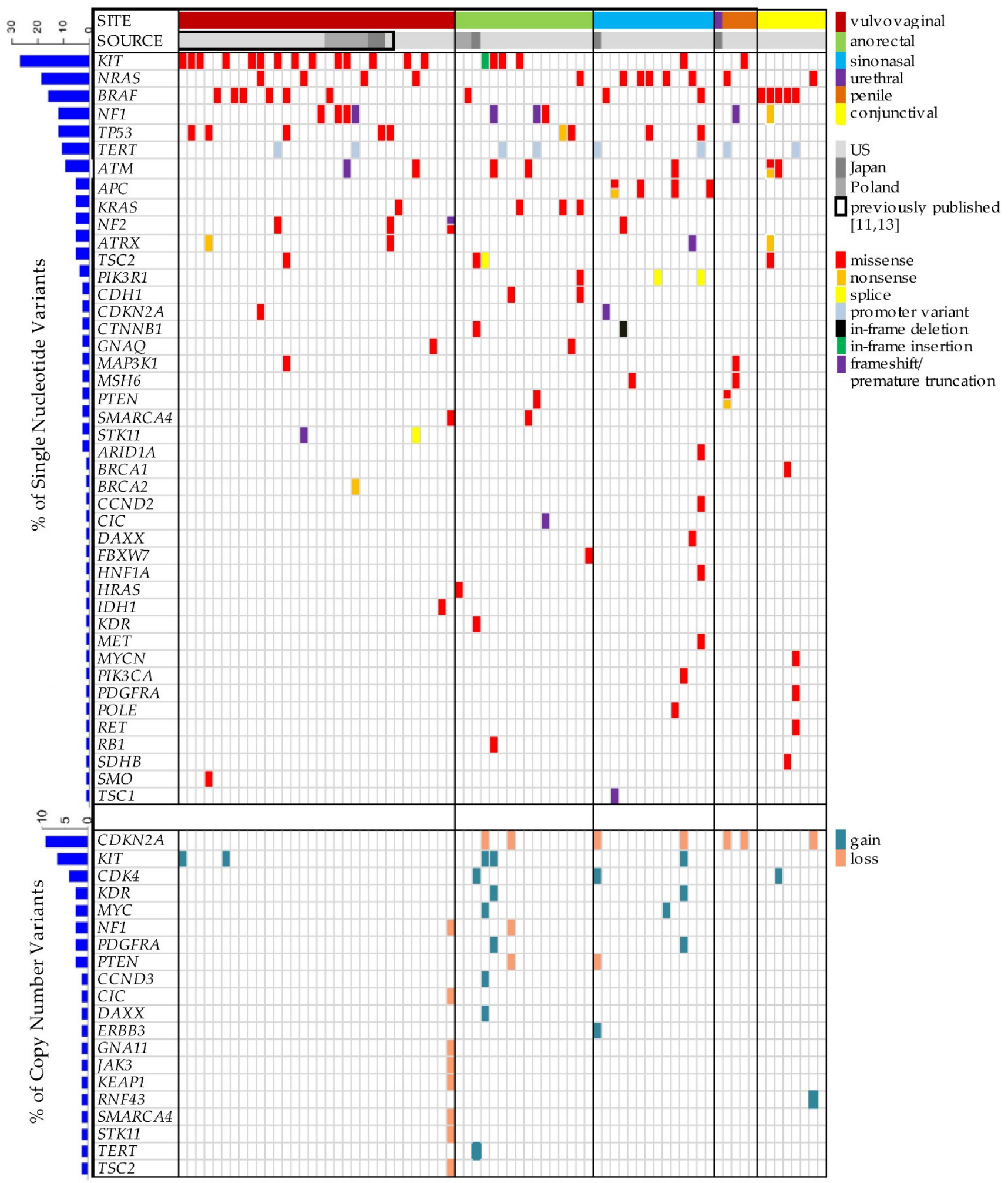

Figure 3. Summary of next-generation sequencing performed on 75 cases. 

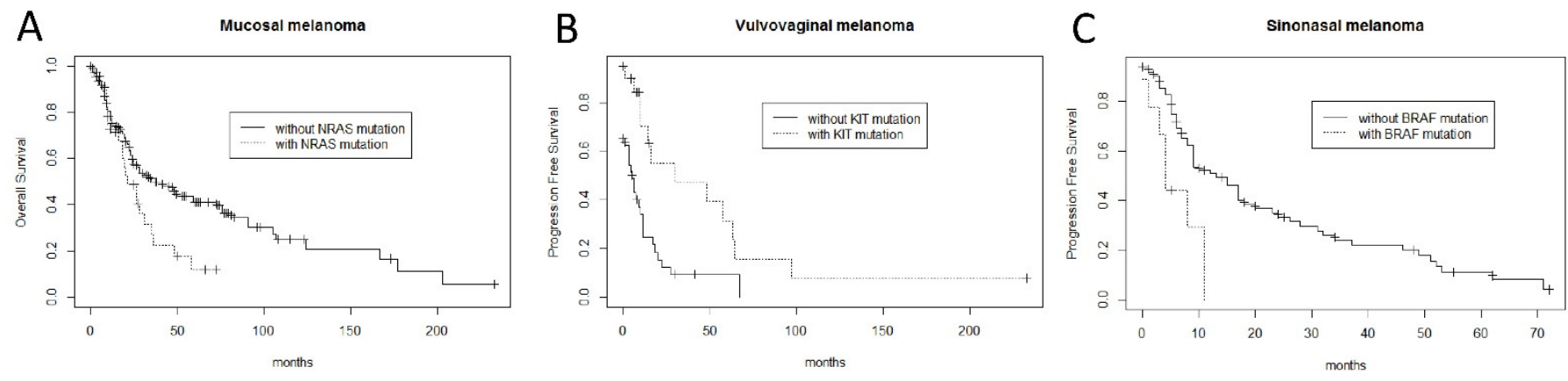

Figure 4. Kaplan-Meier curves demonstrate significant correlation between: (A) worse overall survival and NRAS mutation in mucosal melanomas (log-rank $p=0.028$ ); (B) better progression-free survival and KIT mutation in vulvovaginal melanomas $(\log$-rank $p=0.0021)$; $(\mathbf{C})$ worse progression-free survival and BRAF mutation in sinonasal melanomas (log-rank $p=0.0045)$.

In multivariate analyses, NRAS mutation remained as an independent predictor of worse OS ( $p=0.036)$ and worse MSS $(p=0.024)$. Higher stage ( 3 or 4$)$ at diagnosis remained as independent predictor of worse OS as well as MSS ( $p=0.026$ and 0.0012 , respectively) (Table 2).

Table 2. Multivariate Cox proportional hazards models.

\begin{tabular}{ccccc}
\hline & \multicolumn{2}{c}{ Overall Survival } & \multicolumn{2}{c}{ Melanoma-Specific Survival } \\
\cline { 2 - 5 } & Hazard Ratio & $p$-Value & Hazard Ratio & $p$-Value \\
\hline NRAS mutation & 1.71 & $0.036^{*}$ & 1.80 & $0.024^{*}$ \\
\hline Stage (3-4 versus 1-2) & 1.71 & $0.026^{*}$ & 2.11 & $0.0012 *$ \\
\hline Age (> 65 years) & 1.41 & 0.10 & - & - \\
\hline Ulceration & 1.49 & 0.11 & - & - \\
\hline${ }^{*} p<0.05$, statistical significance. & & & &
\end{tabular}

The percentages of NRAS, BRAF, KIT, IGF2R and SF3B1 mutations in different geographic regions are summarized in Table S5. BRAF mutation was more frequent in cases from Europe and Asia $(p=0.0066)$. NRAS mutation was detected more frequent in cases from North America versus Asia $(p=0.035)$.

\section{Discussion}

Mucosal melanomas are a rare and aggressive disease associated with frequent recurrence and distant metastases. The poor prognosis is likely a result of delay in diagnosis due to anatomic location. Overall survival (OS) has been cited to be highest in the vulvovaginal melanoma group, followed by sinonasal melanoma, and then anorectal melanoma $[4,8,20]$. Similar to findings reported in a series of 706 mucosal melanomas by Cui et al. [6], no significant correlation between survival and anatomic sites is observed in our study. Male gender, older age, depth of tumor, presence of ulceration and advanced stage are reported unfavorable prognostic variables [4,6,8,20-23]. In a series of 444 mucosal melanomas from a European population head and neck location, male gender, advanced tumor stage, nodal disease and incomplete resection status were independent risk factors for disease progression [8]. Older age and advanced stage are worse prognostic parameters in a German series of 161 patients [4]. In an analysis of 644 patients with vulvar melanoma, age less than 68 was an independent predictor of improved OS [24]. On the contrary, in some prior studies, age had no prognostic significance [6,25-27]. In our study, only advanced stage at diagnosis remains an independent negative prognosticator in multivariate analyses.

Geographic region might have a role in survival differences, and further studies are needed. We observe different frequencies of BRAF and NRAS mutations in cases 
from North America and Europe than from Asia. Similar differences in distribution of genetic variants between Western countries and Asia have been also reported in cutaneous melanomas [28]. Patients from Europe have significantly better OS $(p=0.01)$ in comparison to those from North America and Asia in our study. The reason is not known and further investigation is needed.

The prognostic role of histologic features in mucosal melanomas remains uncertain. In a study of 706 patients with mucosal melanomas by Lian et al. [29], depth of tumor invasion, number of lymph node metastases and distant metastases were independent prognosticators for OS in multivariate analyses and were similar for different mucosal sites. However, thickness has not been shown to be a predictor of survival in an analysis of 1824 mucosal melanomas since it cannot be determined in majority of mucosal melanomas due to the fragmented nature of the specimens and tangential nature of the histologic sections [22].

Dermal mitotic rate $\left(\geq 2 / \mathrm{mm}^{2}\right)$ has been shown to be an unfavorable prognosticator in vulvar and vulvovaginal melanomas $[27,30]$. On the contrary, mitotic rate had no significant impact on survival in a study of 86 mucosal melanoma patients by Cinotti et al. [23] and in another study of 85 cases by Tcheung et al. [31]. Similarly, we do not observe increased mitotic activity to be significantly associated with reduced survival in mucosal melanoma patients in our series.

The presence of ulceration correlates with worse OS in univariate analyses in our series. Similarly, Heppt et al. [8] showed that presence of ulceration is an important predictor of shorter OS. On the contrary, ulceration has no prognostic significance for OS in a series of 706 prospectively-followed patients with mucosal melanoma [6]. There is a trend toward significance for worse PFS and lymphovascular invasion in our study. Keller et al. [32] observed that lymphovascular invasion was strongly correlated with decreased survival in mucosal melanoma patients. In a series of 46 patients with anorectal melanoma, the presence of perineural invasion was identified as an independent predictor of disease-specific mortality in multivariate analysis [33].

The Mitogen-Activated Protein Kinase (MAPK) pathway plays an important role in melanoma pathogenesis. V-raf murine sarcoma viral oncogene homolog $\mathrm{B}(B R A F)$ and neuroblastoma RAS viral oncogene homolog (NRAS) mutations are different in mucosal melanomas in comparison to cutaneous melanomas [34]. NRAS mutant melanomas (NEMOs) are reported to be associated with increased risk of visceral and central nervous system metastases in comparison to wild-type cutaneous tumors [35]. The presence of NRAS mutations correlated with worse OS in a series of 2793 cutaneous melanomas by Bai and colleagues [36]. Similarly, we observe that NRAS mutation correlated with worse OS and MSS in mucosal melanomas. Although mutations at codon 61 are seen in both cutaneous and mucosal melanomas, mutations involving codons 12 and 13 (G13D, G12A and G12D) occur more frequently in mucosal melanomas [34]. Dumaz et al. [34] reported NRAS mutations in $12 \%(179 / 1454)$ of mucosal melanomas with $54 \%(96 / 179)$ and $46 \%$ (83/179) located on Q61 and G12/G13, respectively. Similarly, we observe NRAS mutations in $19 \%(37 / 198)$ with $62 \%(23 / 37)$ on codon Q61 and $38 \%(14 / 37)$ on codon G12/13.

Melanomas that harbor NRAS mutation, either previously untreated and those progressed on immunotherapy, might be targeted by MEK inhibitor such as Binimetinib or Pimasertib $[37,38]$. In melanoma cell lines with activating NRAS mutations, combination of PI3K or AKT inhibitors with MEK inhibitors has demonstrated synergistic inhibition [39]. For patients with NRAS mutant melanomas, there have been several clinical trials of combination therapy: combination of CDK4/6 inhibitor (LEE011) and MEK inhibitor (MEK162) in phase $\mathrm{Ib} / \mathrm{II}$ clinical trial [40], and combination of RAF inhibitor (LXH254) with ERK1/2 inhibitor (LTT462) or Trametinib (MEK inhibitor) in phase Ib clinical trial [41].

$B R A F$ mutations have been reported in $8 \%(107 / 1339)$ of mucosal melanoma involving $\mathrm{V} 600 \mathrm{E}$ in $63 \%(67 / 107)$ and another codon in the remaining $37 \%(40 / 107)$ [34]. In line with published results, we observe $B R A F$ mutations in $12 \%(25 / 201)$ of studied mucosal melanomas with V600E and variants detected in $72 \%$ and $28 \%$, respectively. In conventional 
melanoma, BRAF-mutated tumors have been reported to be more aggressive than the corresponding wild-type tumors [42]. Although no correlation with prognosis is observed for all mucosal melanomas in the current series, BRAF mutation correlates with worse PFS for sinonasal melanomas when subgroups are analyzed. V600E mutation is observed in mucosal melanomas involving half of the conjunctival melanomas in our study [43]. D594G, G469A and K601E are the frequently observed BRAF variants in mucosal melanomas [34]. Similarly, A581S, L579Q, G469R, D594G and G466V BRAF variants are detected in our series. BRAF inhibitors have been shown to significantly lengthen PFS and OS in patients with melanoma harboring BRAF V600 mutations (V600E and V600K) [42,44]. However, BRAF inhibitors target tumors harboring BRAF V600E and not BRAF variants; therefore, other treatment modalities such as RAF inhibitor are currently under study [42-45].

Alterations in KIT play an important role in tumor growth, proliferation and metastases in a variety of cancer [46]. KIT mutations in primary melanoma are composed of missense substitutions on different exon distribution in comparison to KIT-mutated gastrointestinal stromal tumor [47]. KIT mutation has been reported from $10 \%$ to $21 \%$ of mucosal melanomas $[47,48]$. The overall KIT alteration frequency of $19 \%$ to $39 \%$, with the L576P mutation being the most common mutation and KIT amplification seen in 1026\% [47,48]. Patients whose tumors harbor KIT L576P and K642E mutations can be targeted with KIT inhibitors such as imatinib, sunitinib, dasatinib and nilotinib [42]. While KIT mutation and/or amplification were reported to be adverse prognostic marker in melanomas in the Asian population, KIT mutation correlates with better PFS for vulvar melanomas in our series $[49,50]$. It could be that a large percentage of acral melanoma was included in these published series.

SF3B1 (splicing factor 3 subunit B1) mutation has been reported to be associated with good prognosis as well as late metastases in different series of uveal melanomas [51,52]. SF3B1, a mutation of codon 625 seen in $84 \%$ of the cases, does not correlate with prognosis in our series of mucosal melanomas. Recurrent R625C and R625H mutations are the main mutations reported in uveal, vulvovaginal and anorectal melanomas [2,51,52]. While Newell et al. [5] reported SF3B1 mutations most frequently in mucosal melanomas from Europe, we observe no geographical differences for SF3B1 in our study.

In a recent study by Iida et al. [10], the IGF $2 R$ variants were detected in $32 \%$ of cases, making it the most commonly mutated gene in mucosal melanoma. Our results do not confirm the high frequency of highly pathogenic IGF $2 R$ variants, with $18 \%$ of cases harbored the mutation. Although IGF2R L252V mutation is frequently detected in our study (data not shown), it is most likely benign polymorphism, reported in almost $14 \%$ of population worldwide [53]. Excluding the low and moderate pathogenic variants from our analysis and focusing only on highly pathogenic variants may be the reason of observed differences in IGF2R mutation frequency between ours and study by Iida et al. [10]. Although our results do not confirm the direct role of $I G F 2 R$ mutations in driving mucosal melanoma development, there are data suggesting its more complex role. As shown in cutaneous melanoma, reduced expression of IGF2R inhibits the metastatic potential of melanoma cells [54]. Lately, it has been shown that the IGF axis with an emphasis on the IGF2R gene is responsible for metastatic niche formation by transforming the normal fibroblast into cancer-associated fibroblasts (CAFs) [55]. These results suggest that IGF2R variants may have a complex, yet so far unknown role in driving mucosal melanoma progression, for which an explanation requires further research.

Recent studies of mucosal melanomas by whole exome sequencing demonstrated that mucosal melanomas have a low mutational burden, with frequent structural variants commonly affecting CDK4, MDM2 and TERT [5,9]. From the available NGS data of our cases, copy number variants affecting CDKN2A and KIT are frequently seen in mucosal melanomas. In addition to commonly noted $B R A F, N R A S$ and $K I T$, mutations affecting the APC, ATM, ATRX, CDH1, KRAS, NF1, NF2, PIK3R1, TSC2, TP53 and TERT promoter regions are noted. When the mucosal melanoma is triple (BRAF, NRAS, NF1)-negative, KIT is the most commonly mutated gene in vulvovaginal melanomas, while APC and KRAS 
are detected mainly in sinonasal and anorectal melanomas, respectively. Co-mutation of KIT and NF1, previously reported by Hintzsche et al. [56] and seen in 2 vulvar melanomas of our prior study [11], was detected in one anorectal melanoma in the current series. Although SPRED1, HLA-A and CHD8 are not included in our NGS panel, well-known driver genes of melanomas such as KRAS, NF1, SF3B1, TP53 and TERT are detected in our mucosal melanoma cases.

Our study has several limitations. Due to the multicenter nature of our study, the patients included in our study did not receive uniform surgical and/or medical treatment. A high failure rate was observed with next-generation sequencing tests performed on old archival materials. Nevertheless, our study includes a significant number of these rare subtypes of melanomas for analyses.

\section{Conclusions}

In conclusion, our series of mucosal melanomas confirms frequent mutation of melanoma driver genes, including BRAF, NRAS, KIT, KRAS, SF3B1, NF1, TP53 and TERT. In multivariate analyses NRAS mutation remains a predictor of worse survival independent of stage in mucosal melanomas. KIT mutation correlates with improved PFS for vulvovaginal melanomas and $B R A F$ mutation with worse PFS for sinonasal melanomas only in univariate analyses. The significance of frequently mutated IGF2R in mucosal melanomas remains unclear. There appears to be some geographical differences in molecular alterations; however, larger cohorts of mucosal melanomas are needed for further investigation.

Supplementary Materials: The following are available online at https:/ / www.mdpi.com/article / 10.3390/cells10092216/s1, Table S1: Primers for polymerase chain reaction amplification and sequencing. Table S2: IGF2R methods. Table S3: The single nucleotide variants and indel gene targets covered by the next-generation sequencing tests are as follows (exons). Table S4: Univariate Cox proportional hazards models. Table S5: Geographic distribution of NRAS, BRAF, KIT, SF3B1 and IGF2R mutations.

Author Contributions: Conceptualization and supervision, M.P.H.; funding acquisition, J.P.W., A.M. and M.P.H.; methodology, J.P.W., A.U., D.D.-S. and M.P.H.; acquisition, analysis and interpretation of data, J.P.W., A.U., D.D.-S., C.-L.W., M.F., M.A.S., W.B., J.R., A.M. and M.P.H.; technical and material support J.P.W., A.U., D.D.-S., C.-L.W., M.F., M.A.S., W.B., J.R., A.M. and M.P.H.; writing original draft, J.P.W. and M.P.H.; review and edit manuscript, J.P.W., A.U., D.D.-S., C.-L.W., M.F., M.A.S., W.B., J.R., A.M. and M.P.H. All authors have read and agreed to the published version of the manuscript.

Funding: A Greater Poland Cancer Centre intramural grant, no: 2/2019 (206) (11/01/2019/ZP/WCO/002) was awarded to J.P.W.

Institutional Review Board Statement: This study was conducted according to the guidelines of the Declaration of Helsinki and approved by the Partners Human Research Committee, the institutional review board of Partners HealthCare (protocol code 2011P0001665, date of approval: 1 July 2019).

Informed Consent Statement: Patient consent was waived since the study was performed on preexisting archived pathology specimens. No patients were involved.

Data Availability Statement: All data generated or analyzed during this study are included in this published article and its supplementary information files.

Acknowledgments: We thank the following colleagues for contributing cases to our prior published studies, the results of which were included in the current study: Agata Chlopik, Poznan University Medical Sciences and Greater Poland Cancer Center, Poznan, Poland; Maria-Teresa FernandezFigueras, Hospital Universitari Germans Trias i Pujol, Univeristat Autònoma de Barcelona, Barcelona, Spain; Yan Peng and Jason Mull, University of Texas Southwestern Medical Center, Dallas, TX, USA; Kristen Paral and Christopher R. Shea, University of Chicago, IL, USA; Gemma Tell-Marti and Susana Puig, Hospital Clínic de Barcelona, IDIBAPS, and Centre of Biomedical Research on Rare Diseases (CIBERER), ISCIII, Barcelona, Spain; Sara C. Shalin, University of Arkansas, Little Rock, AR, USA.

Conflicts of Interest: The authors declare no conflict of interest. 


\section{References}

1. Chang, A.E.; Karnell, L.H.; Menck, H.R. The National Cancer Data Base report on cutaneous and noncutaneous melanoma: A summary of 84,836 cases from the past decade. The American College of Surgeons Commission on Cancer and the American Cancer Society. Cancer 1998, 83, 1664-1678. [CrossRef]

2. Yang, H.M.; Hsiao, S.J.; Schaeffer, D.F.; Lai, C.; Remotti, H.E.; Horst, D.; Mansukhani, M.M.; Horst, B.A. Identification of recurrent mutational events in anorectal melanoma. Mod. Pathol. 2017, 30, 286-296. [CrossRef]

3. Hayward, N.K.; Wilmott, J.S.; Waddell, N.; Johansson, P.A.; Field, M.A.; Nones, K.; Patch, A.M.; Kakavand, H.; Alexandrov, L.B.; Burke, H.; et al. Whole-genome landscapes of major melanoma subtypes. Nature 2017, 545, 175-180. [CrossRef] [PubMed]

4. Sarac, E.; Amaral, T.; Keim, U.; Leiter, U.; Forschner, A.; Eigentler, T.K.; Garbe, C. Prognostic factors in 161 patients with mucosal melanoma: A study of German Central Malignant Melanoma Registry. J. Eur. Acad. Dermatol. Venereol. 2020, 34, $2021-2025$. [CrossRef] [PubMed]

5. Newell, F.; Kong, Y.; Wilmott, J.S.; Johansson, P.A.; Ferguson, P.M.; Cui, C.; Li, Z.; Kazakoff, S.H.; Burke, H.; Dodds, T.J.; et al. Whole-genome landscape of mucosal melanoma reveals diverse drivers and therapeutic targets. Nat. Commun. 2019, $10,3163$. [CrossRef]

6. Cui, C.; Lian, B.; Zhou, L.; Song, X.; Zhang, X.; Wu, D.; Chi, Z.; Si, L.; Sheng, X.; Kong, Y.; et al. Multifactorial analysis of prognostic factors and survival rates among 706 mucosal melanoma patients. Ann. Surg. Oncol. 2018, 25, 2184-2192. [CrossRef]

7. Amit, M.; Tam, S.; Abdelmeguid, A.S.; Roberts, D.B.; Takahashi, Y.; Raza, S.M.; Su, S.Y.; Kupferman, M.E.; DeMonte, F.; Hanna, E.Y. Mutation status among patients with sinonasal mucosal melanoma and its impact on survival. Br. J. Cancer 2017, 116, $1564-1571$. [CrossRef] [PubMed]

8. Heppt, M.V.; Roesch, A.; Weide, B.; Gutzmer, R.; Meier, F.; Loquai, C.; Kähler, K.C.; Gesierich, A.; Meissner, M.; von Bubnoff, D.; et al. Prognostic factors and treatment outcomes in 444 patients with mucosal melanoma. Eur. J. Cancer 2017, 81, 36-44. [CrossRef]

9. Zhou, R.; Shi, C.; Tao, W.; Li, J.; Wu, J.; Han, Y.; Yang, G.; Gu, Z.; Xu, S.; Wang, Y.; et al. Analysis of mucosal melanoma whole-genome landscapes reveals clinically relevant genomic aberrations. Clin. Cancer Res. 2019, 25, 3548-3560. [CrossRef]

10. Iida, Y.; Salomon, M.P.; Hata, K.; Tran, K.; Ohe, S.; Griffiths, C.F.; Hsu, S.C.; Nelson, N.; Hoon, D.S.B. Predominance of triple wild-type and IGF2R mutations in mucosal melanomas. BMC Cancer 2018, 18, 1054. [CrossRef] [PubMed]

11. Dias-Santagata, D.; Selim, M.A.; Su, Y.; Peng, Y.; Vollmer, R.; Chłopik, A.; Tell-Marti, G.; Paral, K.M.; Shalin, S.C.; Shea, C.R.; et al. KIT mutations and CD117 overexpression are markers of better progression-free survival in vulvar melanomas. Br. J. Dermatol. 2017, 177, 1376-1384. [CrossRef] [PubMed]

12. Wroblewska, J.P.; Mull, J.; Wu, C.L.; Fujimoto, M.; Ogawa, T.; Marszalek, A.; Hoang, M.P. SF3B1, NRAS, KIT, and BRAF mutation; CD117 and cMYC expression; and tumoral pigmentation in sinonasal melanomas: An analysis with newly found molecular alterations and some population-based molecular differences. Am. J. Surg. Pathol. 2019, 43, 168-177. [CrossRef] [PubMed]

13. Moran, J.M.T.; Fujimoto, M.; Iwahashi, Y.; Cornejo, K.M.; Hoang, M.P. Molecular alterations in vaginal melanomas: Report of 4 cases and literature review. Am. J. Dermatopathol. 2021, 43, 45-48. [CrossRef]

14. Li, H.; Durbin, R. Fast and accurate short read alignment with Burrows-Wheeler transform. Bioinformatics 2009, 25, 1754-1760. [CrossRef]

15. Van der Auwera, G.A.; Carneiro, M.O.; Hartl, C.; Poplin, R.; Del Angel, G.; Levy-Moonshine, A.; Jordan, T.; Shakir, K.; Roazen, D.; Thibault, J.; et al. From FastQ data to high confidence variant calls: The Genome Analysis Toolkit best practices pipeline. Curr. Protoc. Bioinform. 2013, 43, 11.10.1-11.10.33. [CrossRef]

16. Cingolani, P.; Patel, V.M.; Coon, M.; Nguyen, T.; Land, S.J.; Ruden, D.M.; Lu, X. Using Drosophila melanogaster as a model for genotoxic chemical mutational studies with a new program, SnpSift. Front. Genet. 2012, 3, 35. [CrossRef]

17. Zheng, Z.; Liebers, M.; Zhelyazkova, B.; Cao, Y.; Panditi, D.; Lynch, K.D.; Chen, J.; Robinson, H.E.; Shim, H.S.; Chmielecki, J.; et al. Anchored multiplex PCR for targeted next-generation sequencing. Nat. Med. 2014, 20, 1479-1484. [CrossRef]

18. Kent, W.J. BLAT-The BLAST-like alignment tool. Genome Res. 2002, 12, 656-664. [CrossRef]

19. R Development Core Team. R: A Language and Environment for Statistical Computing. Available online: http:/ /www.R-project. org / (accessed on 5 November 2020).

20. Schaefer, T.; Satzger, I.; Gutzmer, R. Clinics, prognosis and new therapeutic options in patients with mucosal melanoma: A retrospective analysis of 75 patients. Medicine 2017, 96, e5753. [CrossRef]

21. Hahn, H.M.; Lee, K.G.; Choi, W.; Cheong, S.H.; Myung, K.B.; Hahn, H.J. An updated review of mucosal melanoma: Survival meta-analysis. Mol. Clin. Oncol. 2019, 11, 116-126. [CrossRef] [PubMed]

22. Altieri, L.; Eguchi, M.; Peng, D.H.; Cockburn, M. Predictors of mucosal melanoma survival in a population-based setting. J. Am. Acad. Dermatol. 2019, 81, 136-142.e2. [CrossRef] [PubMed]

23. Cinotti, E.; Chevallier, J.; Labeille, B.; Cambazard, F.; Thomas, L.; Balme, B.; Leccia, M.T.; D'Incan, M.; Vercherin, P.; Douchet, C.; et al. Mucosal melanoma: Clinical, histological and c-kit gene mutational profile of 86 French cases. J. Eur. Acad. Dermatol. Venereol 2017, 31, 1834-1840. [CrossRef] [PubMed]

24. Sugiyama, V.E.; Chan, J.K.; Shin, J.Y.; Berek, J.S.; Osann, K.; Kapp, D.S. Vulvar melanoma: A multivariable analysis of 644 patients. Obstet. Gynecol. 2007, 110, 296-301. [CrossRef] [PubMed]

25. Sinasac, S.E.; Petrella, T.M.; Rouzbahman, M.; Sade, S.; Ghazarian, D.; Vicus, D. Melanoma of the vulva and vagina: Surgical management and outcomes based on a clinicopathologic review of 68 cases. J. Obstet. Gynaecol. Can. 2019, 41, 762-771. [CrossRef] 
26. Gru, A.A.; Becker, N.; Dehner, L.P.; Pfeifer, J.D. Mucosal melanoma: Correlation of clinicopathologic, prognostic, and molecular features. Melanoma Res. 2014, 24, 360-370. [CrossRef]

27. Seifried, S.; Haydu, L.E.; Quinn, M.J.; Scolyer, R.A.; Stretch, J.R.; Thompson, J.F. Melanoma of the vulva and vagina: Principles of staging and their relevance to management based on a clinicopathologic analysis of 85 cases. Ann. Surg. Oncol. 2015, 22, 1959-1966. [CrossRef]

28. Luo, Y.; Zhang, Z.; Liu, J.; Li, L.; Xu, X.; Yao, X.; Dai, Z.; Wang, X.; Yang, S.; Wu, H.; et al. Characterizations of gene alterations in melanoma patients from Chinese population. BioMed Res. Int. 2020, 2020, 6096814. [CrossRef]

29. Lian, B.; Cui, C.L.; Zhou, L.; Song, X.; Zhang, X.S.; Wu, D.; Si, L.; Chi, Z.H.; Sheng, X.N.; Mao, L.L.; et al. The natural history and patterns of metastases from mucosal melanoma: An analysis of 706 prospectively-followed patients. Ann. Oncol. 2017, 28, 868-873. [CrossRef]

30. Nagarajan, P.; Curry, J.L.; Ning, J.; Piao, J.; Torres-Cabala, C.A.; Aung, P.P.; Ivan, D.; Ross, M.I.; Levenback, C.F.; Frumovitz, M.; et al. Tumor thickness and mitotic rate robustly predict melanoma-specific survival in patients with primary vulvar melanoma: A retrospective review of 100 cases. Clin. Cancer Res. 2017, 23, 2093-2104. [CrossRef]

31. Tcheung, W.J.; Selim, M.A.; Herndon, J.E., II; Abernethy, A.P.; Nelson, K.C. Clinicopathologic study of 85 cases of melanoma of the female genitalia. J. Am. Acad. Dermatol. 2012, 67, 598-605. [CrossRef] [PubMed]

32. Keller, D.S.; Thomay, A.A.; Gaughan, J.; Olszanski, A.; Wu, H.; Berger, A.C.; Farma, J.M. Outcomes in patients with mucosal melanomas. J. Surg. Oncol. 2013, 108, 516-520. [CrossRef] [PubMed]

33. Yeh, J.J.; Shia, J.; Hwu, W.J.; Busam, K.J.; Paty, P.B.; Guillem, J.G.; Coit, D.G.; Wong, W.D.; Weiser, M.R. The role of abdominoperineal resection as surgical therapy for anorectal melanoma. Ann. Surg. 2006, 244, 1012-1017. [CrossRef] [PubMed]

34. Dumaz, N.; Jouenne, F.; Delyon, J.; Mourah, S.; Bensussan, A.; Lebbé, C. Atypical BRAF and NRAS mutations in mucosal melanoma. Cancers 2019, 11, 1133. [CrossRef]

35. Adler, N.R.; Wolfe, R.; Kelly, J.W.; Haydon, A.; McArthur, G.A.; McLean, C.A.; Mar, V.J. Tumour mutation status and sites of metastasis in patients with cutaneous melanoma. Br. J. Cancer 2017, 117, 1026-1035. [CrossRef]

36. Bai, X.; Kong, Y.; Chi, Z.; Sheng, X.; Cui, C.; Wang, X.; Mao, L.; Tang, B.; Li, S.; Lian, B.; et al. MAPK Pathway and TERT promoter gene mutation pattern and its prognostic value in melanoma patients: A retrospective study of 2,793 cases. Clin. Cancer Res. 2017, 23, 6120-6127. [CrossRef]

37. Dummer, R.; Schadendorf, D.; Ascierto, P.A.; Arance, A.; Dutriaux, C.; Di Giacomo, A.M.; Rutkowski, P.; Del Vecchio, M.; Gutzmer, R.; Mandala, M.; et al. Binimetinib versus dacarbazine in patients with advanced NRAS-mutant melanoma (NEMO): A multicentre, open-label, randomised, phase 3 trial. Lancet Oncol. 2017, 18, 435-445. [CrossRef]

38. Lebbé, C.; Dutriaux, C.; Lesimple, T.; Kruit, W.; Kerger, J.; Thomas, L.; Guillot, B.; Braud, F.; Garbe, C.; Grob, J.J.; et al. Pimasertib versus Dacarbazine in patients with unresectable NRAS-mutated cutaneous melanoma: Phase II, randomized, controlled trial with crossover. Cancers 2020, 12, 1727. [CrossRef]

39. Greger, J.G.; Eastman, S.D.; Zhang, V.; Bleam, M.R.; Hughes, A.M.; Smitheman, K.N.; Dickerson, S.H.; Laquerre, S.G.; Liu, L.; Gilmer, T.M. Combinations of BRAF, MEK, and PI3K/mTOR inhibitors overcome acquired resistance to the BRAF inhibitor GSK2118436 dabrafenib, mediated by NRAS or MEK mutations. Mol. Cancer Ther. 2012, 11, 909-920. [CrossRef] [PubMed]

40. ClinicalTrials.gov. A Phase Ib/II Study of LEE011 in Combination with MEK162 in Patients with NRAS Mutant Melanoma (NCT01781572). Available online: https:/ / clinicaltrials.gov/ct2/show / NCT01781572 (accessed on 22 August 2021).

41. ClinicalTrials.gov. A Phase Ib Study of LXH254-Centric Combinations in NSCLC or Melanoma (NCT02974725). Available online: https: / / clinicaltrials.gov/ct2/show / NCT02974725 (accessed on 22 August 2021).

42. Long, G.V.; Hauschild, A.; Santinami, M.; Atkinson, V.; Mandalà, M.; Chiarion-Sileni, V.; Larkin, J.; Nyakas, M.; Dutriaux, C.; Haydon, A.; et al. Adjuvant Dabrafenib plus Trametinib in stage III BRAF-mutated melanoma. N. Engl. J. Med. 2017, 377, 1813-1823. [CrossRef]

43. Brouwer, N.J.; Verdijk, R.M.; Heegaard, S.; Marinkovic, M.; Esmaeli, B.; Jager, M.J. Conjunctival melanoma: New insights in tumour genetics and immunology, leading to new therapeutic options. Prog. Retin. Eye Res. 2021, 17, 100971. [CrossRef] [PubMed]

44. Dummer, R.; Ascierto, P.A.; Gogas, H.J.; Arance, A.; Mandala, M.; Liszkay, G.; Garbe, C.; Schadendorf, D.; Krajsova, I.; Gutzmer, R.; et al. Encorafenib plus binimetinib versus vemurafenib or encorafenib in patients with BRAF-mutant melanoma (COLUMBUS): A multicentre, open-label, randomised phase 3 trial. Lancet Oncol. 2018, 19, 603-615. [CrossRef]

45. Okimoto, R.A.; Lin, L.; Olivas, V.; Chan, E.; Markegard, E.; Rymar, A.; Neel, D.; Chen, X.; Hemmati, G.; Bollag, G.; et al. Preclinical efficacy of a RAF inhibitor that evades paradoxical MAPK pathway activation in protein kinase BRAF-mutant lung cancer. Proc. Natl. Acad. Sci. USA 2016, 113, 13456-13461. [CrossRef] [PubMed]

46. Meng, D.; Carvajal, R.D. KIT as an oncogenic driver in melanoma: An update on clinical development. Am. J. Clin. Dermatol. 2019, 20, 315-323. [CrossRef]

47. Carvajal, R.D.; Antonescu, C.R.; Wolchok, J.D.; Chapman, P.B.; Roman, R.A.; Teitcher, J.; Panageas, K.S.; Busam, K.J.; Chmielowski, B.; Lutzky, J.; et al. KIT as a therapeutic target in metastatic melanoma. JAMA 2011, 305, 2327-2334. [CrossRef]

48. Curtin, J.A.; Busam, K.; Pinkel, D.; Bastian, B.C. Somatic activation of KIT in distinct subtypes of melanoma. J. Clin. Oncol. 2006, 24, 4340-4346. [CrossRef]

49. Kong, Y.; Si, L.; Zhu, Y.; Xu, X.; Corless, C.L.; Flaherty, K.T.; Li, L.; Li, H.; Sheng, X.; Cui, C.; et al. Large-scale analysis of KIT aberrations in Chinese patients with melanoma. Clin. Cancer Res. 2011, 17, 1684-1691. [CrossRef] [PubMed] 
50. Jin, S.A.; Chun, S.M.; Choi, Y.D.; Kweon, S.S.; Jung, S.T.; Shim, H.J.; Yun, S.J. BRAF mutations and KIT aberrations and their clinicopathological correlation in 202 Korean melanomas. J. Investig. Dermatol. 2013, 133, 579-582. [CrossRef]

51. Furney, S.J.; Pedersen, M.; Gentien, D.; Dumont, A.G.; Rapinat, A.; Desjardins, L.; Turajlic, S.; Piperno-Neumann, S.; de la Grange, P.; Roman-Roman, S.; et al. SF3B1 mutations are associated with alternative splicing in uveal melanoma. Cancer Discov. 2013, 3, 1122-1129. [CrossRef] [PubMed]

52. Yavuzyigitoglu, S.; Koopmans, A.E.; Verdijk, R.M.; Vaarwater, J.; Eussen, B.; van Bodegom, A.; Paridaens, D.; Kilic, E.; de Klein, A.; Rotterdam Ocular Melanoma Study Group. Uveal melanomas with SF3B1 mutations: A distinct subclass associated with lateonset metastases. Ophthalmology 2016, 123, 1118-1128. [CrossRef]

53. Karczewski, K.J.; Francioli, L.C.; Tiao, G.; Cummings, B.B.; Alföldi, J.; Wang, Q.; Collins, R.L.; Laricchia, K.M.; Ganna, A.; Birnbaum, D.P.; et al. The mutational constraint spectrum quantified from variation in 141,456 humans. Nature 2020, 581, 434-443. [CrossRef]

54. Levy, C.; Khaled, M.; Iliopoulos, D.; Janas, M.M.; Schubert, S.; Pinner, S.; Chen, P.H.; Li, S.; Fletcher, A.L.; Yokoyama, S.; et al. Intronic miR-211 assumes the tumor suppressive function of its host gene in melanoma. Mol. Cell 2010, 40, 841-849. [CrossRef] [PubMed]

55. Dror, S.; Sander, L.; Schwartz, H.; Sheinboim, D.; Barzilai, A.; Dishon, Y.; Apcher, S.; Golan, T.; Greenberger, S.; Barshack, I.; et al. Melanoma miRNA trafficking controls tumour primary niche formation. Nat. Cell Biol. 2016, 18, 1006-1017. [CrossRef] [PubMed]

56. Hintzsche, J.D.; Gorden, N.T.; Amato, C.M.; Kim, J.; Wuensch, K.E.; Robinson, S.E.; Applegate, A.J.; Couts, K.L.; Medina, T.M.; Wells, K.R.; et al. Whole-exome sequencing identifies recurrent SF3B1 R625 mutation and comutation of NF1 and KIT in mucosal melanoma. Melanoma Res. 2017, 27, 189-199. [CrossRef] [PubMed] 